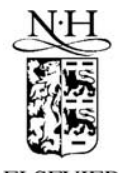

ELSEVIER mathematical

social

sciences

Mathematical Social Sciences 43 (2002) 405-428

www.elsevier.com/locate/econbase

\title{
A general concept of majority rule
}

\author{
Michel Regenwetter ${ }^{\mathrm{a}, *}$, A.A.J. Marley ${ }^{\mathrm{b}}$, Bernard Grofman ${ }^{\mathrm{c}}$ \\ ${ }^{a}$ Department of Psychology, University of Illinois at Urbana-Champaign, 603 East Daniel Street, \\ Champaign, IL 61820, USA \\ ${ }^{\mathrm{b}}$ Department of Psychology, McGill University, Montreal, PQ, Canada \\ ${ }^{\mathrm{c}}$ School of Social Sciences, University of California at Irvine, Irvine, CA, USA
}

\begin{abstract}
We develop a general concept of majority rule for finitely many choice alternatives that is consistent with arbitrary binary preference relations, real-valued utility functions, probability distributions over binary preference relations, and random utility representations. The underlying framework is applicable to virtually any type of choice, rating, or ranking data, not just the linear orders or paired comparisons assumed by classic majority rule social welfare functions. Our general definition of majority rule for arbitrary binary relations contains the standard definition for linear orders as a special case.
\end{abstract}

(C) 2002 Elsevier Science B.V. All rights reserved.

Keywords: Condorcet criterion; Majority rule; Partial orders; Random utility; Semiorders; Weak orders

\section{Introduction}

Much of the work in social choice theory on the topic of preference aggregation involves one or more of the following restrictions on the domain of study.

Firstly, beginning with Arrow's Social Choice and Individual Values (Arrow, 1951), social choice theories have tended to posit that actors have linear order or weak order preferences. More general binary preference relations have largely been ignored.

Secondly, until recently, much of the work posited deterministic preferences rather than allowing for probabilistic preferences, including relative frequencies of deterministic preferences as a special case of a probability measure over preference relations.

*Corresponding author. Tel.: +1-217-333-0763; fax: +1-217-244-5876.

E-mail address: regenwet@uiuc.edu (M. Regenwetter).

${ }^{1}$ However, there are some notable exceptions, including the literature on probabilistic voting schemes (Coughlin, 1992; Enelow and Hinich, 1984, 1989; Fishburn and Gehrlein, 1977; Fishburn, 1975, 1984; Intriligator, 1973; Schofield and Tovey, 1992). 
Thirdly, to our knowledge, the social choice literature has not treated the various deterministic and probabilistic representations of preferences and their relationship to various deterministic and probabilistic representations of utility in a unifying way. For instance, very few social choice results have been explicitly stated in terms of random utility representations. ${ }^{2}$

In this paper we focus on one particularly important preference aggregation mechanism, namely majority rule. Our general concept of majority rule (with respect to a finite number of choice alternatives) is applicable to all binary preference relations, real-valued utility functions, probability distributions over binary preference relations, and random utility representations. The definition of majority rule for binary preferences has the following simple form. For a profile of $n$ many binary preference relations (possibly with repetitions), alternative $\mathrm{A}$ is majority preferred to alternative $\mathrm{B}$ if and only if the total number of preference relations in which $\mathrm{A}$ is preferred to $\mathrm{B}$ exceeds the total number of preference relations in which B is preferred to A. Much of the social choice literature has dealt with deterministic preferences. Because probability distributions over binary relations contain relative frequencies/tallies as a special case, we make use of a probabilistic framework. Here, given a probability distribution over binary preference relations, alternative A beats B by a majority if and only if the total probability of those relations in which $\mathrm{A}$ is 'better than' $\mathrm{B}$ exceeds the total probability of those relations in which B is 'better than' A.

There are several reasons for developing a definition of majority rule that is fully general in the way specified above. Firstly, it is desirable to have a concept of majority rule that is general enough to be well defined in a random utility modeling framework. Secondly, mathematical generality is obviously desirable in and of itself, e.g. all binary relations, not just linear and weak orders, should be accounted for. Thirdly, the literature on preference and utility provides considerable evidence that actual preferences are much more general than linear or weak orders and discusses a panoply of utility representations (Fishburn, 1970; Kahneman and Tversky, 1979; Loomes and Sugden, 1982; Luce, 1959; Luce and von Winterfeldt, 1994; Luce and Suppes, 1965; Tversky, 1969; Tversky and Kahneman, 1986). Indeed, sometimes this literature suggests that individual preference need not even be transitive (Tversky, 1969; Gehrlein, 1989; Vila, 1998). Fourthly, most ballot data or preferences recorded in public opinion surveys do not explicitly require or record all, or even any, pairwise comparisons. Thus, if we wish to reconstruct majority preferences from empirical data, we need a general framework which permits such inferences. This paper provides exactly such a framework. In an empirical companion paper, using approval voting and feeling thermometer survey data, we demonstrate that the inferred majority preferences may critically depend on the assumed form of the preference or utility representation.

\footnotetext{
${ }^{2}$ Although there are, again, noteworthy exceptions (Falmagne and Regenwetter, 1996; Regenwetter, 1997; Tangian, 2000).

${ }^{3}$ This includes the possibilities of cycles at the individual preference level. Considering just the pair A,B the cycle 'A preferred to B preferred to $\mathrm{C}$ preferred to A' would be tallied for A 'better than' $\mathrm{B}$, and the reverse cycle 'A preferred to C preferred to B preferred to A' would be tallied for B 'better than' A.
} 
The paper has four more sections. Section 2 reviews the most commonly used (deterministic) binary preference relations.

Section 3 offers a general concept of majority rule in terms of binary preference relations. We begin that section with a definition of net preference probability, which is key to most of the subsequent theorems. The net preference probability of a binary relation is equal to the probability of the binary relation minus the probability of the binary relation that is obtained by reversing all pairs in the first relation. For instance, the net preference probability, which we sometimes abbreviate net probability, of a ranking ' $a$ best, $b$ second, $c$ worst', is the probability of the ranking ' $a$ best, $b$ second, $c$ worst' minus the probability of the ranking ' $c$ best, $b$ second, $a$ worst'. We use the idea of net preference probabilities to define majority rule social welfare relations for finitely many choice alternatives in a way that is consistent with all binary preference relations, and probability distributions over such binary preference relations. Since most of the existing literature focuses on linear order preferences, we provide the only possible way to translate a net probability distribution on general binary relations over triples into a net probability distribution over linear orders on the same triples that preserves pairwise net preference probabilities (Theorem 1). This theorem essentially allows us to translate tallies on (probability distributions on) binary relations into tallies on (probability distributions on) linear orders in a fashion that preserves majority rule.

Section 4 discusses random utility representations. We begin by summarizing known results on deterministic real-valued representations of various types of binary preference relations (Theorem 2). We also review previous work on the corresponding relationship between probability distributions over preference relations, on the one hand, and random utilities, on the other (Theorem 3). We extend the existing results by adding new theorems on a random utility representation for strict partial orders of dimension less than some number $k$ (Theorems 4 and 5). As a consequence of these representations, our general definition of majority rule in terms of preference relations is naturally extended to utility functions and random utility representations (Observations 1 and 2).

In Section 5, we comment on how to generalize the impartial culture and related cultures of indifference to our general framework (Observation 3).

\section{Preference relations}

Most commonly used representations of preference take the form of binary (order) relations. Here we present results for linear orders, strict weak orders, semiorders, interval orders, strict partial orders of dimension $\leq k$, and even arbitrary binary relations on finitely many choice alternatives. ${ }^{4}$ We state most of our results in terms of arbitrary binary relations (which we project down to the family of asymmetric binary relations, with a mathematical 'trick' and without loss of generality for our results on majority

\footnotetext{
${ }^{4}$ As noted earlier, theorems on social choice are often stated only in terms of linear orders or (strict) weak orders. A noteworthy exception is Brady and Ansolabehere (1989) who discuss a palette of representations ranging from linear orders to the family of all transitively closed binary relations.
} 
rule). Of course, because the various types of orders are special cases of binary relations, the results apply as a consequence to them as well.

Throughout the paper we focus on a basic finite set $\mathscr{C}$ of choice alternatives (commodities, political candidates) and we denote $|\mathscr{C}|$ by $N$. A binary relation $B$ on $\mathscr{C}$ is a set $B \subseteq \mathscr{C}^{2}$. For $(x, y) \in B$, we also write $x B y$ (as in ' $x$ is better than $y$ '). Given a binary relation $B$, we write $B^{-1}=\{(y, x) \mid x B y\}$ and $\bar{B}=\mathscr{C}^{2}-B$. The relative product $B B^{\prime}$ of two binary relations $B$ and $B^{\prime}$ is the binary relation $\left\{(x, z) \in \mathscr{C}^{2} \mid \exists y \in \mathscr{C}\right.$ s.t. $\left.x B y B^{\prime} z\right\} . I=\{(x, x) \mid x \in \mathscr{C}\}$ denotes the identity relation on $\mathscr{C}$. A strict partial order $B$ on $\mathscr{C}$ is an asymmetric (i.e. $B \cap B^{-1}=\emptyset$ ), transitive (i.e. $B B \subseteq B$ ) binary relation.

An interval order is a strict partial order $B$ with the additional property that $B B^{-1} B \subseteq B$ (see, for instance, Roberts, 1979). The key idea behind interval orders (Fishburn, 1970, 1985) is that we cannot always distinguish between choice alternatives that are very similar. Therefore, interval orders capture the idea that an object is preferred to another if and only if it is sufficiently superior to the other. The threshold of distinction may depend on the objects that are being compared, i.e. it may be variable. A semiorder is an interval order with a fixed threshold (Luce, 1956, 1959), the latter being captured by the additional property that $B B B^{-1} \subseteq B$ (see, for instance, Roberts, 1979). A strict weak order is an asymmetric and negatively transitive (i.e. $\bar{B} \bar{B} \subseteq \bar{B}$ ) binary relation. A linear order is a strict weak order without ties, in other words, a weakly connected (i.e. $\bar{I} \subseteq B \cup B^{-1}$ ) strict weak order. When the set $\mathscr{C}$ is given, we use the notation $\Pi$ to refer to the collection of all linear orders over $\mathscr{b}$ and $\pi$ for a given linear order. There is a one-to-one correspondence between linear orders and what is commonly referred to as 'rankings without ties'. We will use the two terms interchangeably. A weak order is a connected (i.e. $B \cup B^{-1}=\mathscr{C}^{2}$ ), transitive binary relation.

It is well known that each strict partial order can be written as an intersection of linear orders. The dimension of a strict partial order is the smallest number $k$ such that the strict partial order is the intersection of $k$ many linear orders. Dimension theory is a thriving research domain, for instance Trotter (1992) provides a detailed analysis. In addition to their use in combinatorics and operations research, strict partial orders are also studied in much detail in the mathematical social sciences. For instance, Falmagne (1997) and others develop stochastic processes on (strict) partial orders to study the evolution of preferences over time. Regenwetter et al. (1999) successfully analyze a national election panel with two time points using strict weak orders. There is also continued interest in (strict) partial orders in economic theory (see, Duggan (1999) for a recent example).

\section{A general concept of majority rule}

By covering all binary relations, we are able to derive the existence or nonexistence of majority rule transitive social welfare orders even when many members of a population

\footnotetext{
${ }^{5}$ We will not go into the detailed semantic distinctions between strict and weak individual preference, or between indifference and incomparability, because these distinctions do not affect majority rule outcomes.
} 
have cyclic (individual) preferences. In other words, we allow for the possibility that society may be 'rational' even when many individuals are not.

As already pointed out, majority rule is usually defined in terms of hypothetical pairwise competitions of each candidate against each of the others. In contrast to the conventional definition, virtually no social choice procedure explicitly requires or records pairwise comparisons or complete rankings. Therefore, following the same logic as Regenwetter and Grofman (1998a,b), we will redefine majority rule in terms of different primitives: the distribution of (possibly unobservable) utilities or preferences in the population at large. Indeed, a problem neglected by many empirical analyses of voting and social choice in political science is that of statistical identifiability. One of our priorities here is to define majority rule in such a way that we can treat the problem of finding a majority winner in a manner general enough to be compatible with a statistical sampling and inference framework. In the empirical companion paper, we demonstrate how such inferences can crucially depend on the underlying model specifications. Furthermore, a separate line of work (Regenwetter et al., 2002a; Tsetlin and Regenwetter, 2002) tackles the statistical issues raised by the sampling and inference framework.

In majority rule, what really matters is whether $\mathrm{C}$ is preferred to $\mathrm{D}$ more often than $\mathrm{D}$ is preferred to $\mathrm{C}$, or vice versa. Motivated by this observation, Regenwetter and Grofman (1998a,b) study so-called net preference probabilities for linear orders, which we now formally define for arbitrary binary preference relations. Similar deterministic concepts are discussed in Feld and Grofman (1986), Gaertner and Heinecke (1978), and Saari (1995).

Definition 1. A probability distribution on a collection $\mathscr{B}$ of binary relations is a mapping $P: \mathscr{B} \rightarrow[0,1]$ where $B \mapsto P(B)$ and $\Sigma_{B \in \mathscr{B}} P(B)=1$. Writing, as before, $B^{-1}=$ $\{(b, a) \mid a B b\}$ for the reverse of $B$, and given a probability distribution $P$ on a collection $\mathscr{B}$ of binary relations, the net preference probability (on $\mathscr{B}$ ) of $B \in \mathscr{B}, N P^{\mathscr{B}}(B)$, is given by

$$
N P^{\mathscr{B}}(B)= \begin{cases}P(B)-P\left(B^{-1}\right) & \text { if } B^{-1} \in \mathscr{B}, \\ P(B) & \text { otherwise. }\end{cases}
$$

The mapping $N P^{\mathscr{B}}: \mathscr{B} \rightarrow[-1,1]$ is a net (preference) probability distribution. The binary net preference probability of (the preference of) $a$ over $b$ is

$$
N P_{a b}^{\mathscr{B}}=\sum_{\substack{B \in \mathscr{B} \\ a B b}} N P^{\mathscr{B}}(B) .
$$

(The empty sum is assumed to be equal to zero.)

In order to be able to state our results succinctly for many different classes of relations at once, we will use the following convention (mathematical 'trick').

Definition 2. Consider any probability distribution $P$ on a collection $\mathscr{B}$ of binary relations on $\mathscr{C}$ and the corresponding net probability distribution $N P^{\mathscr{B}}$ defined in Definition 1. Writing $A S(D)=D-\left(D \cap D^{-1}\right)$ for the asymmetric part of a binary 
relation $D$, define the net probability distribution $N P$ (without the superscript) on the set of all asymmetric binary relations on $\mathscr{C}$ as follows: for any asymmetric binary relation $B \subseteq \mathscr{C}^{2}$ (not necessarily in $\mathscr{B}$ )

$$
N P(B)=\sum_{\substack{D \in \mathscr{B} \\ B=A S(D)}} N P^{\mathscr{B}}(D),
$$

where an empty sum is set to be zero. The corresponding binary net preference probability $N P_{a b}$ of (the preference of) $a$ over $b$ is given by

$$
N P_{a b}=\sum_{\substack{B \in \mathscr{B} \\ a B b}} N P(B) .
$$

Notice that with this definition $N P_{a b}=N P_{a b}^{\mathscr{B}}$. In other words, (1) we embed the collection of any linear orders, strict weak orders, semiorders, interval orders, strict partial orders naturally in the collection of all asymmetric binary relations, and (2) we collapse all binary relations into their asymmetric part because relationships of the form $a B a$ are not relevant for binary net preference probabilities, and relationships of the form $a B b B a$ cancel out with themselves in the binary net preference probabilities. In particular, whenever we refer to a probability distribution on binary relations, we can, without loss of generality for majority rule outcomes, assume that the probabilities of symmetric relations (i.e. relations $B$ such that $B^{-1}=B$ ) or reflexive relations (i.e. relations $B$ such that $I \subseteq B$ ) are zero, since we can project those relations down to their asymmetric parts. ${ }^{6}$ This is the convention being used whenever we leave out the superscript $\mathscr{B}$ from $N P^{\mathscr{B}}$. Some authors distinguish ' $a B b$ and $b B a$ ' (often referred to as 'indifference' between $a$ and $b$ ) from 'neither $a B b$ nor $b B a$ ' (often referred to as 'incomparability' of $a$ and $b$ ). We do not go into these issues here, because they are inconsequential to the analysis of majority preferences.

We now proceed to provide a general definition of majority rule based on a set $\mathscr{B}$ of arbitrary binary preference relations. (In order to be able to keep track of particular cases, we state the definition with the superscript.) Grandmont (1978) develops somewhat related concepts.

\footnotetext{
${ }^{6}$ For instance, the asymmetric part of a symmetric relation is the empty set, i.e. the strict weak order representing complete indifference between the choice alternatives. More interestingly, the asymmetric part of the (reflexive) weak order $\{(a, a),(a, b),(b, b),(b, c),(c, c),(a, c)\}$ on the set $\{a, b, c\}$ is the strict weak order $\{(a, b),(b, c),(a, c)\}$ on the set $\{a, b, c\}$.

${ }^{7}$ Suppose that $2 / 3$ of the population has the weak order $\{(a, a),(b, b),(c, c),(a, b),(a, c),(b, c),(c, b)\}$ and the remaining $1 / 3$ of the population has the weak preference order $\{(a, a),(b, b),(c, c),(b, c),(c, a),(b, a)\}$. Using Definition 2, we will treat that situation as though $2 / 3$ of the population had the strict weak order preference $\{(a, b),(a, c)\}$ and the remaining $1 / 3$ the strict weak order preference $\{(b, c),(c, a),(b, a)\}$. So, for instance, ' $b B c$ and $c B b^{\prime}$ ' is replaced by 'neither $b B c$ nor $c B b$ ' in the preference relation held by $2 / 3$ of the population. The majority preference relation, with the indifference relationships excluded, is $\{(a, b),(b, c),(a, c)\}$, which, we believe, is also the only reasonable definition of the majority relation for the original distribution (with the indifferences included). In other words, the indifference relationships cancel out in majority rule calculations.
} 
Definition 3. Given a probability distribution $P$ on a set $\mathscr{B}$ of binary relations over a finite set $\mathscr{C}$ and given the corresponding net preference probability distribution $N P^{\mathscr{B}}$, define majority rule social welfare relations $\succeq$ and $>$ through, $\forall c, d \in \mathscr{C}$,

$$
\begin{aligned}
& c \succeq d \Leftrightarrow N P_{c d}^{\mathscr{B}} \geq 0, \\
& c>d \Leftrightarrow N P_{c d}^{\mathscr{B}}>0 .
\end{aligned}
$$

We say that $c$ has a weak majority over $d$ (including the possibility of a tie) whenever $c \succeq d$. We also say that $c$ has a strict majority over $d$ (excluding the possibility of a tie) whenever $c>d$.

In words, $a$ has a strict majority over $b$ if $a$ is preferred to $b$ strictly more often (or with more total probability mass) than $b$ is preferred to $a$.

Some notation for various individual preference relations is in order. Suppose for a moment that $\mathscr{C}=\{a, b, c\}$. We write

$a$
$b$
$c$

for the binary relation $\{(a, b),(a, c),(b, c)\}$, a linear order. We write

$$
\begin{gathered}
a \\
b c
\end{gathered}
$$

for the binary relation $\{(a, b),(a, c)\}$, a strict weak order in which $b$ and $c$ are tied and both are dominated by $a$. Similarly, we write

$$
b c
$$$$
a
$$

for the binary relation $\{(b, a),(c, a)\}$, a strict weak order in which $b$ and $c$ are tied and both dominate $a$. The symbol

$a$
$b$

stands for the binary relation $\{(a, b)\}$, a strict partial order (which is also an interval order, and, in fact a semiorder) where $a$ and $c$ are tied, and also $c$ and $b$ are tied, while $a$ is strictly preferred to $b$. The symbol

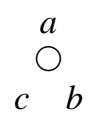

stands for a (forward, clockwise) cycle $\{(a, b),(b, c),(c, a)\}$. The symbol

$$
\begin{aligned}
& a \\
& c
\end{aligned}
$$

denotes the nontransitive binary relation (read clockwise) $\{(a, b),(b, c)\}$. Finally, $a b c$ denotes the state of total indifference between the three choice alternatives, i.e. the empty relation $\emptyset$. Up to relabeling of the choice alternatives, the above six relations are all possible asymmetric binary relations on three choice alternatives, which makes a total of 27 different relations. 
Because the conditions involved in the study of social orders, namely transitivity and negative transitivity, are conditions on triples, we now investigate various properties for triples of choice alternatives. Note that every strict partial order on three choice alternatives is in fact also a semiorder and an interval order. There are 19 different strict partial orders on three choice alternatives, 13 of which are strict weak orders, of which in turn six are linear orders. Notice also that we always have $N P(a b c)=0$. Thus, going from the most general to the most specific binary relations, the net preference probability distribution is positive on at most 13 binary relations, nine semiorders, six strict weak orders, three linear orders.

Definition 4. Given a net preference probability distribution $N P^{\mathscr{B}}$ (associated with some probability distribution $P$ ) on a set $\mathscr{B}$ of binary relations over a finite set $\mathscr{C}$, if $|\mathscr{C}| \geq 3$ and $\{a, b, c\} \subset \mathscr{C}$, then the marginal net preference probabilities over $\{a, b, c\}$ are defined as follows: for $B^{\prime} \subseteq\{a, b, c\}^{2}$

$$
N P^{\mathscr{B}}\left(B^{\prime}\right)=\sum_{\substack{B \in \mathscr{B} \\ B \cap\{a, b, c\}^{2}=B^{\prime}}} N P^{\mathscr{B}}(B) .
$$

The next theorem and its corollaries show how the general case of majority rule tallies on binary relations, strict partial orders, semiorders, interval orders and strict weak orders can be translated into equivalent majority rule tallies on linear orders. We use the fact that, for any strict partial order $B$ over a set $\mathscr{C}$ and for any triple $\{x, y, z\} \subseteq \mathscr{C}$, the binary relation $B \cap\{x, y, z\}^{2}$ is a semiorder. We also continue to use the convention (of Definition 2) that net probabilities $N P(B)$ are defined only on asymmetric binary relations $B$.

Theorem 1. Let NP be a net probability distribution (associated with some probability distribution $P$ ) on the set of all asymmetric binary relations over $\mathscr{C}$. Let $N P^{\Pi}$ be a net probability distribution (associated with some probability distribution $P^{\Pi}$ ) on the set $\Pi$ of all linear orders over $\mathscr{C}$ and let $\mathscr{C}^{\prime} \subseteq \mathscr{C}$ with $\left|\mathscr{C}^{\prime}\right|=3$. Then the identity

$$
N P_{x y}^{\Pi}=N P_{x y}, \quad \forall x, y \in \mathscr{C}^{\prime}, x \neq y,
$$

holds if and only if

$$
\begin{aligned}
& N P^{\Pi}\left(\begin{array}{l}
a \\
b \\
c
\end{array}\right)=N P\left(\begin{array}{l}
a \\
b \\
c
\end{array}\right)
\end{aligned}
$$

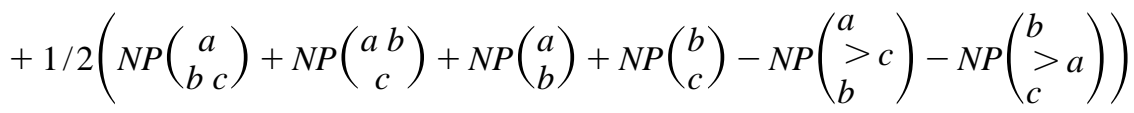

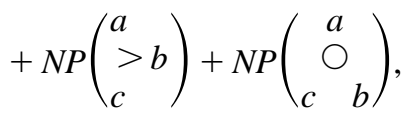

for all relabelings $\{a, b, c\}=\mathscr{C}^{\prime}$. Suppose that (7) holds for all three-element subsets $\mathscr{C}^{\prime} \subseteq \mathscr{C}$. Then, $\geq$ as defined in Definition 3 agrees for NP and NP ${ }^{I I}$. The same holds for $>$. 
The proof is provided in Appendix A.

Obvious special cases occur when individual preferences are all strict partial orders (semiorders, interval orders) or all strict weak orders. We present the cases for strict partial orders (Corollary 1) and strict weak orders (Corollary 2).

Corollary 1. Let $N P^{\mathscr{P O}}$ be a net probability distribution (associated with some probability distribution $P^{\mathscr{P O}}$ ) on the set $\mathscr{P} \mathscr{O}$ of all strict partial orders over $\mathscr{C}$ and let $N P^{\Pi}$ be a net probability distribution (associated with some probability distribution $P^{\Pi}$ ) on the set $\Pi$ of all linear orders over $\mathscr{C}$. Let $\mathscr{C}^{\prime} \subseteq \mathscr{C}$ with $\left|\mathscr{C}^{\prime}\right|=3$. Then the identity

$$
N P_{x y}^{\Pi}=N P_{x y}^{\mathscr{P O}}, \quad \forall x, y \in \mathscr{C}^{\prime}, x \neq y
$$

holds if and only if, for all relabelings $\{a, b, c\}=\mathscr{C}^{\prime}$,

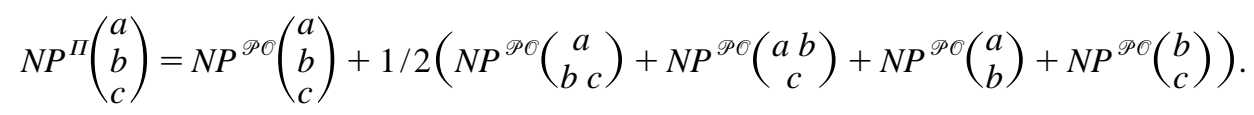

Suppose that (9) holds for all three element subsets $\mathscr{C}^{\prime} \subseteq \mathscr{C}$. Then, $\succeq$ as defined in Definition 3 agrees for $N P^{\Pi}$ and $N P^{\mathscr{P O}}$. The same holds for $>$.

A parallel corollary holds with $\mathscr{P} \odot$ replaced by the set $\mathscr{W} \odot$ of strict weak orders on $\mathscr{C}$.

Corollary 2. Let $N P^{\mathscr{W O}}$ be a net probability distribution (associated with some probability distribution $P^{\mathscr{W O}}$ ) on the set $\mathscr{W} \mathscr{O}$ of all strict weak orders over $\mathscr{C}$ and let $N P^{I}$ be a net probability distribution (associated with some probability distribution $P^{I I}$ ) on the set $\Pi$ of all linear orders over $\mathscr{C}$. Let $\mathscr{C}^{\prime} \subseteq \mathscr{C}$ with $\left|\mathscr{C}^{\prime}\right|=3$. Then the identity

$$
N P_{x y}^{\Pi}=N P_{x y}^{\mathscr{W O}} \quad \forall x, y \in \mathscr{C}^{\prime}, x \neq y,
$$

holds if and only if, for all relabelings $\{a, b, c\}=\mathscr{C}^{\prime}$,

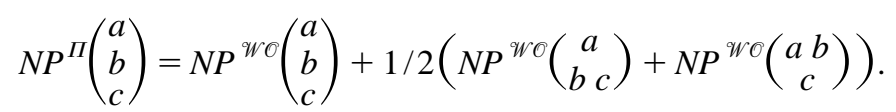

Suppose that (11) holds for all three element subsets $\mathscr{C}^{\prime} \subseteq \mathscr{C}$. Then, $\succeq$ as defined in Definition 3 agrees for $N P^{\Pi}$ and NP ${ }^{\mathscr{W O}}$. The same holds for $>$.

The proofs of the two corollaries are straightforward.

\section{Majority rule based on utility functions or random utility representations}

In this section we move from preference relations to utility functions. We first discuss the relationship between preference representations and utility representations, both deterministic and probabilistic. Then we define a general concept of majority rule in terms of utility functions and random utility representations. We subsequently show how 
majority rule defined in terms of preference representations relates to majority rule defined in terms of utility representations.

Most mathematical representations of utility rely on real-valued functions that map objects into their utility values. A very general conceptual framework to represent and quantify the variability of utilities is provided by random utility theory. Here, the utility of an object is (the value of) a random variable (or a random vector). Just as preference relations are in close correspondence with utility functions from $\mathscr{C}$ into $\mathbb{R}^{n}$ (for some $n$ ), probability distributions over preference relations are in close correspondence with random utilities. Just as probability distributions over preference relations generalize and include deterministic preference relations, so do random utilities generalize and include real-valued deterministic utility functions. Note that we make no assumptions about where the randomness comes from. Probabilities may capture random error, random sampling, probabilistic mechanisms inside the decision-maker's head, or they may simply quantify the 'proportion' of the population which satisfies some property. In particular, we require no independence assumptions.

Since people interact and communicate we do not impose the impartial culture (uniform distribution) assumption but instead allow interindividual preferences to be interdependent and/or systematically biased in the following sense: in a probability distribution over preference relations, interdependencies can simply be quantified through setting the probabilities of certain preference orders very high or very low; in the random utility framework, the interdependent nature of utilities is captured and quantified through the joint distribution of the utility random variables. We also make no assumptions about that joint distribution. We first review deterministic representations and then discuss random utility representations.

There is a close relationship between preference orders and real valued utility functions. This well-known relationship (in the deterministic realm) has been spelled out in representation theorems of the following kind (for references, see for instance, Fishburn, 1985; Krantz et al., 1971; Roberts, 1979). (Note that these formulations hold only for finite $\mathscr{C}$. The infinite case is more complex.)

Theorem 2. Let $B$ be a binary relation on a finite set $\mathscr{C} . B$ is a strict weak order if and only if it has a real representation $u: \mathscr{C} \rightarrow \mathbb{R}$ of the following form:

$$
a B b \Leftrightarrow u(a)>u(b) .
$$

If $B$ is a linear order, then it has the above representation, but the converse holds only if $u$ is a one-to-one mapping. $B$ is a semiorder if and only if it has a real representation $u: \mathscr{b} \rightarrow \mathbb{R}$ of the following form:

$$
a B b \Leftrightarrow u(a)>u(b)+\epsilon
$$

where $\epsilon$ is a fixed (utility) threshold. $B$ is an interval order if and only if it has a real representation $l, u: \mathscr{C} \rightarrow \mathbb{R}$, with $l(x)<u(x)$ (for all $x$ ), of the following form:

$$
a B b \Leftrightarrow l(a)>u(b) .
$$

In the interval order representation, the utility functions $u$ and $l$ can be interpreted as 
upper and lower utility, respectively, and thus the function $(u-l)$ can be interpreted as an object-dependent utility threshold.

We now move on to variable preferences and utilities. There is no particular reason why individual preference orders should not vary and why the utility of a commodity should not differ for different observers. A natural and general way to account for and quantify variability is through probability measures. We can simply introduce a probability distribution over binary preference relations of a given type, as we have seen already. Alternatively, we can define the utility of an object to be a random variable. (For ease of reading, random variables will be written in boldface.)

Definition 5. A random utility representation on a finite set $\mathscr{C}$ of choice alternatives is a collection of jointly distributed real valued random variables $\boldsymbol{U}=\left(\boldsymbol{U}_{i, c}\right)_{i=1, \ldots, k ; c \in \mathscr{C}}$ (with a finite $k$ ) on some sample space with probability measure $\mathbb{P}$. Given a measurable set $X \subseteq \mathbb{R}^{|\mathscr{C}|}$, we write $\mathbb{P}(\boldsymbol{U} \in X)$ for the probability that the joint utility takes a value in $X$.

We will always use the same symbol $\mathbb{P}$ for the probability measure on the sample space underlying a random utility representation, since this does not lead to any ambiguities. For instance, we can interpret the probability $\mathbb{P}(\boldsymbol{U} \in X)$ as the proportion of people in the population who give the candidates joint utilities in $X$. In particular, if $X=\left\{\left(x_{c}\right)_{c \in \mathscr{C}} \in \mathbb{R}^{\mathscr{C}} \mid x_{d}=x_{e} ; \forall d, e \in \mathscr{C}\right\}$, and $k=1$, then $\mathbb{P}(\boldsymbol{U} \in X)$ can for instance be interpreted as the proportion of voters who are completely indifferent between all candidates, in the sense that they assign equal utilities to all of them. Another interpretation, in a sampling framework, would be that $\mathbb{P}(\boldsymbol{U} \in X)$ (with the above choice of $X$ ) is the probability that a randomly selected voter is completely indifferent between all candidates.

In order to situate our approach, several remarks are in order. See Fishburn (1998) for an excellent overview of the related literature.

1. Much traditional research in random utility modeling (Ben-Akiva and Lerman, 1985; McFadden, 1991, 1998; Thurstone, 1927a,b) places all randomness in an error term by assuming the following form:

$$
\boldsymbol{U}=U+\boldsymbol{E},
$$

where $U$ is a real vector (interpreted as the true utility of the objects in $\mathscr{C}$ ) and $\boldsymbol{E}$ is a family of jointly distributed random variables (interpreted as a vector of random errors or exogenous shocks). Furthermore, random utility models often assume that the mean of $\boldsymbol{E}$ is zero, and/or that $\boldsymbol{E}$ belongs to a particular parametric family, typically either the multivariate normal (Thurstone, 1927a,b) or the multivariate extreme value family (Joe, 1997; McFadden, 1998). While this scenario is a special case of our framework, we will generally not make any assumptions about the joint distribution of the family $\boldsymbol{U}$. Furthermore, we emphasize that randomness has many substantive interpretations, including error only as a special case. In fact, for the 
study of social choice, the interpretation of random utility as true value plus noise is not very useful, interesting or realistic. The purpose of social choice theory is not to eliminate random error, but rather to aggregate truly variable preferences via a social welfare function.

2. The research on nonparametric random utility models is quite extensive and has focused mainly on the characterization problems of probabilistic binary choice (Fishburn, 1992; Koppen, 1995; Marley, 1991; Marschak, 1960; Suck, 1992, 1995) and of probabilistic multiple choice (Barberá, 1979; Barberá and Pattanaik, 1986; Falmagne, 1978). The interpretation of our family $\boldsymbol{U}$ of jointly distributed utility random variables is simply that utilities of objects vary and co-vary. The distribution of utility values together with their interdependencies is formally captured by the joint distribution of the relevant random variables.

3. As far as probabilistic (preference) relations are concerned, there also exists a literature on parametric models here, for example the work by Critchlow et al. (1991, 1993), Mallows (1957) and Marley (1991).

In sum, we argue that variable preferences can be represented appropriately by a probability distribution over a set of preference relations and variable utilities can be represented appropriately through jointly distributed random variables. The relationship between these two frameworks is reasonably well understood. For instance, Block and Marschak (1960) showed that most families of jointly distributed random variables induce a probability distribution over linear orders (i.e. rankings without ties), and that, conversely, each probability distribution over linear orders can be associated with a (highly nonunique) family of jointly distributed utility random variables. In other words, it is equivalent, for instance, to assume that a randomly sampled observer has a strict linear order preference over all choice alternatives or to assume that $\mathrm{s} / \mathrm{he}$ assigns utilities to objects according to a joint outcome of a family of random variables (satisfying certain properties). Recent developments in mathematical psychology (Niederée and Heyer, 1997; Regenwetter, 1996, 1997; Regenwetter and Marley, 2001; Suck, 1995) have extended this result to arbitrary relations, including the special cases where the individual preferences are transitive binary relations, strict weak orders (i.e. rankings with possible ties), semiorders (Luce, 1956, 1959) — which capture thresholds of utility discrimination — and interval orders (Fishburn, 1970, 1985)—which are a generalization of semiorders to the case with variable thresholds.

The most general framework for discussing random utility representations is distribution-free in that it makes no assumptions about particular parametric families for the underlying distributions. The following theorem summarizes various known results concerning the intimate link between (distribution free) random utilities and (distribution free) probabilistic preference relations. (Detailed citations to the original papers are provided after the theorem statement.) This theorem, as well as the two theorems that follow it, will allow us to have a unified definition of majority rule that applies to (probabilistic) preference representations and (random) utility representations simultaneously.

Theorem 3. A family of jointly distributed real valued utility random variables $\left(\boldsymbol{U}_{c}\right)_{c \in \mathscr{C}}$ satisfies the following properties. 
Random utility representations of linear orders: if noncoincidence holds, that is, if $\mathbb{P}\left(\boldsymbol{U}_{c}=\boldsymbol{U}_{d}\right)=0, \forall c, d \in \mathscr{C}$ then $\mathbb{P}$ induces a probability distribution $\pi \mapsto P(\pi)$ on the set $\Pi$ of linear orders over $\mathscr{C}$ through, for any linear order $\pi=c_{1} c_{2} \ldots c_{N},\left(c_{1}\right.$ is best $, \ldots, c_{N}$ is worst)

$$
P(\pi)=\mathbb{P}\left(\boldsymbol{U}_{c 1}>\boldsymbol{U}_{c 2}>\ldots>\boldsymbol{U}_{c N}\right) .
$$

Random utility representations of weak orders: regardless of the joint distribution of $\boldsymbol{U}, \mathbb{P}$ induces a probability distribution $B \mapsto P(B)$ on the set $\mathscr{W} O$ of strict weak orders over $\mathscr{C}$ throug $h$

$$
P(B)=\mathbb{P}\left(\bigcap_{(a, b) \in B}\left(\boldsymbol{U}_{a}>\boldsymbol{U}_{b}\right) \cap \bigcap_{(c, d) \in \mathscr{C}^{2}-B}\left(\boldsymbol{U}_{c} \leq \boldsymbol{U}_{d}\right)\right) .
$$

Random utility representations of semiorders: regardless of the joint distribution of $\boldsymbol{U}, \mathbb{P}$ induces a probability distribution $B \mapsto P(B)$ on the set $\mathscr{S O}$ of semiorders over $\mathscr{C}$ through, given a threshold $\epsilon \in \mathbb{R}^{++}$,

$$
P(B)=\mathbb{P}\left(\bigcap_{(a, b) \in B}\left(\boldsymbol{U}_{a}>\boldsymbol{U}_{b}+\boldsymbol{\epsilon}\right) \cap \bigcap_{(c, d) \in \mathscr{C}^{2}-B}\left(\boldsymbol{U}_{c}-\boldsymbol{U}_{d} \leq \boldsymbol{\epsilon}\right)\right) .
$$

Random utility representations of interval orders: consider $\boldsymbol{U}=\left(\boldsymbol{U}_{c}\right)_{c \in \mathscr{C}}$ (upper utility), $\boldsymbol{L}=\left(\boldsymbol{L}_{c}\right)_{c \in \mathscr{C}}$ (lower utility), jointly distributed, and such that $\mathbb{P}(\boldsymbol{L} \leq \boldsymbol{U})=1$. Then $\mathbb{P}$ induces a probability distribution $B \mapsto P(B)$ on the set $\mathscr{I} \mathcal{O}$ of interval orders over $\mathscr{C}$ through,

$$
P(B)=\mathbb{P}\left(\bigcap_{(a, b) \in B}\left(\boldsymbol{L}_{a}>\boldsymbol{U}_{b}\right) \cap \bigcap_{(c, d) \in \mathscr{C}^{2}-B}\left(\boldsymbol{L}_{c} \leq \boldsymbol{U}_{d}\right)\right) .
$$

Conversely, each probability distribution on linear orders, strict weak orders, semiorders, or interval orders over $\mathscr{C}$ can be represented in the above fashion (nonuniquely) by a joint distribution of random variables.

The random utility representation of linear orders is the classical result of Block and Marschak (1960) and has been used, e.g. by Falmagne (1978). The generalizations to strict weak orders and semiorders were pointed out by Regenwetter (1996), and the interval order case by Suck (1995) and Regenwetter (1997). Furthermore, Heyer and Niederée (1989, 1992), Niederée and Heyer (1997), Regenwetter (1996), and Regenwetter and Marley (2001) provide general abstract results including binary relations as very special cases. We now add another pair of random utility representation results, which, to our knowledge, have not been previously stated in the literature.

Theorem 4. Let $\mathscr{P O}(k)$ be the collection of all strict partial orders of dimension $\leq k$ over $\mathscr{C}$ (with $k$ finite). Let $\boldsymbol{U}=\left(\boldsymbol{U}_{i, c}\right)_{i=1, \ldots, k ; c \in \mathscr{C}}$ be a family of jointly distributed random variables, and let $\Pi$ denote the set of all linear orders over $\mathscr{C}$. For any strict partial order $B \in \mathscr{P O}(k)$ we write

$$
\Pi^{k}(B)=\left\{\left(\pi_{1}, \pi_{2}, \ldots, \pi_{k}\right) \in \Pi^{k} \mid B=\bigcap_{m=1}^{k} \pi_{m}\right\} .
$$


Then $\boldsymbol{U}$ induces a probability distribution $B \mapsto P(B)$ over $\mathscr{B}$ through

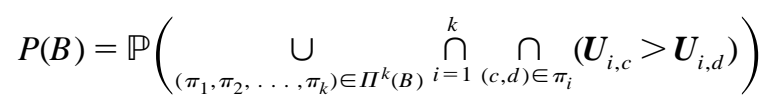

if and only if noncoincidence holds on each component (or dimension) $i$, that is,

$$
\mathbb{P}\left(\boldsymbol{U}_{i, c}=\boldsymbol{U}_{i, d}\right)=0
$$

for $i=1, \ldots, k$, for $(c, d) \in \mathscr{C}^{2}-I$.

The proof is provided in Appendix A. As the next theorem shows, the converse of the above theorem also holds, that is, given a probability distribution over a family of strict partial orders of dimension $\leq k$, we can construct a family of jointly distributed random variables such that (16) is true.

Theorem 5. Consider a probability space of the form $\left\langle\mathscr{P O}(k), 2^{\mathscr{P O}(k)}, P\right\rangle$. Then, for any measurable space $\langle\Omega, \sigma\rangle$, the following two statements are equivalent.

1. There exists a probability measure $\mathbb{P}$ on $\langle\Omega, \sigma\rangle$, and a family $\boldsymbol{U}=\left(\boldsymbol{U}_{i, c}\right)_{i=1, \ldots, k ; c \in \mathscr{C}}$ of jointly distributed random variables on $\langle\Omega, \sigma\rangle$ such that

$$
\mathbb{P}\left(\underset{\left(\pi_{1}, \pi_{2}, \ldots, \pi_{k}\right) \in \Pi^{k}(B)}{\cup} \bigcap_{i=1}^{k} \bigcap_{(c, d) \in \pi_{i}}\left(\boldsymbol{U}_{i, c}>\boldsymbol{U}_{i, d}\right)\right)=P(B) .
$$

2. There are at least as many disjoint nonempty events in $\sigma$ as there are atoms in $\left\langle\mathscr{P} \mathscr{O}(k), 2^{\mathscr{P O}(k)}, P\right\rangle^{8}$

The proof is provided in Appendix A.

We now move on to define pairwise net probabilities for (random) utilities. We start with the situation where we believe that each respondent evaluates the alternatives according to $k$ many different dimensions simultaneously by taking the intersection of $k$ linear orders. (While $k$ is the same for all observers, the actual dimensions may differ.) In other words, we first consider a random utility version of net preference probabilities based on strict partial orders of dimension $\leq k$.

Definition 6. Given jointly distributed random variables $\left(\boldsymbol{U}_{i, c}\right)_{i=1, \ldots, k ; c \in \mathscr{C}}$, the $k$ dimensional binary net (preference) probability of $a$ over $b$ is given by

$$
N P_{a b}=\mathbb{P}\left(\bigcap_{i=1}^{k}\left[\boldsymbol{U}_{i, a}>\boldsymbol{U}_{i, b}\right]\right)-\mathbb{P}\left(\bigcap_{i=1}^{k}\left[\boldsymbol{U}_{i, b}>\boldsymbol{U}_{i, a}\right]\right) .
$$

As the following observation spells out in detail, Definition 1 and Definition 6 yield identical binary net probabilities when the conditions in Theorems 4 and 5 (and thus the resulting relationships) apply.

\footnotetext{
${ }^{8} \mathrm{An}$ atom $\{B\}$ is a single element event which has positive probability.
} 
Observation 1. Let $P$ be a probability distribution over $\mathscr{P} O(k)$ and let $N P_{a b}^{\mathscr{P} O(k)}$ be the pairwise net preference probabilities resulting from Eq. (1) of Definition 1 for that $P$ and for all choices of $a \neq b$ in $\mathscr{C}$. Consider a family $\left(\boldsymbol{U}_{i, c}\right)_{i=1, \ldots, k ; c \in \mathscr{C}}$ of jointly distributed random variables, such that the equalities (16) or (17) hold (with the random variables being noncoincident on each dimension). Then, for all choices of $a \neq b$ in $\mathscr{C}, N P_{a b}$ of Eq. (18) in Definition 6 is identical to $N P_{a b}^{\mathscr{P} \mathscr{O}(k)}$ of Eq. (1) in Definition 1. When $k=1$ and we do not assume noncoincidence, then we get the case when only strict weak orders have nonzero probabilities. Furthermore, the special case of $k=1$, and where noncoincidence holds, yields that only linear orders have nonzero probabilities.

Further special cases of interest are when only semiorders or interval orders (in $\mathscr{P} O(k))$ have nonzero probabilities.

Besides the construction in (18), there is an alternative route for interval orders and semiorders, based on the representation in Theorem 3, which again includes the strict weak orders and linear orders as special cases. Using the random utility representation (15) of interval orders, we can define the net preference probability of $a$ over $b$ in random utility terms as follows.

Definition 7. Given $\left(\boldsymbol{U}_{c}\right)_{c \in \mathscr{A}},\left(\boldsymbol{L}_{c}\right)_{c \in \mathscr{A}}$, jointly distributed random variables with the property that $\mathbb{P}\left(\boldsymbol{U}_{c} \geq \boldsymbol{L}_{c}\right)=1$, the binary net probability (derived from $\boldsymbol{U}, \boldsymbol{L}$ ) of $a$ over $b$ is given by

$$
N P_{a b}=\mathbb{P}\left(\boldsymbol{L}_{a}>\boldsymbol{U}_{b}\right)-\mathbb{P}\left(\boldsymbol{L}_{b}>\boldsymbol{U}_{a}\right) .
$$

Observation 2. Let $P$ be a probability distribution over the set $\mathscr{O} O$ of all interval orders on $\mathscr{C}$ and let $N P_{a b}^{\mathscr{S O}}$ be the pairwise net preference probabilities resulting from Eq. (1) of Definition 1 for that $P$ and for all choices of $a \neq b$ in $\mathscr{b}$.

Let $\left(\boldsymbol{U}_{c}\right)_{c \in \mathscr{A}},\left(\boldsymbol{L}_{c}\right)_{c \in \mathscr{A}}$ be a family of jointly distributed random variables with the property that $\mathbb{P}\left(\boldsymbol{U}_{c} \geq \boldsymbol{L}_{c}\right)=1$ and such that Eq. (15) holds. Then $N P_{a b}$ of Eq. (19) in Definition 7 is identical to $N P_{a b}^{\mathscr{I C}}$ of Eq. (1) in Definition 1 for all choices of $a \neq b$ in $\mathscr{b}$.

Consider the special case where $P$ is a probability distribution over the set $\mathscr{Y O}$ of all semiorders, and where $\boldsymbol{L}_{c}=\boldsymbol{U}_{c}-\boldsymbol{\epsilon}>0$ (everywhere and $\forall c$ ), with $\epsilon \in \mathbb{R}$ a positive constant, i.e. (19) becomes

$$
N P_{a b}=\mathbb{P}\left(\boldsymbol{U}_{a}>\boldsymbol{U}_{b}+\boldsymbol{\epsilon}\right)-\mathbb{P}\left(\boldsymbol{U}_{b}>\boldsymbol{U}_{a}+\boldsymbol{\epsilon}\right) .
$$

If the relation between $P$ and $\mathbb{P}$ given in Eq. (14) holds, then $N P_{a b}$ of Eq. (20) is identical to $N P_{a b}^{\mathscr{C O}}$ of Eq. (1) in Definition 1 for all choices of $a \neq b$ in $\mathscr{b}$.

Consider the next special case where $P$ is a probability distribution over the set $\mathcal{W} O$ of all strict weak orders, and where $\epsilon=0$ in (20), i.e. $\boldsymbol{U}_{c}=\boldsymbol{L}_{c}$ in (19). Then Eq. (20) becomes

$$
N P_{a b}=\mathbb{P}\left(\boldsymbol{U}_{a}>\boldsymbol{U}_{b}\right)-\mathbb{P}\left(\boldsymbol{U}_{b}>\boldsymbol{U}_{a}\right) .
$$

If the relation between $P$ and $\mathbb{P}$ given in Eq. (13) holds, then $N P_{a b}$ of Eq. (21) is identical to $N P_{a b}^{\mathscr{W O}}$ of Eq. (1) in Definition 1 for all choices of $a \neq b$ in $\mathscr{C}$.

Finally, consider the case where $P$ is a probability distribution over the set $\Pi$ of all linear orders, and where noncoincidence is satisfied, that is, $\mathbb{P}\left(\boldsymbol{U}_{c}=\boldsymbol{U}_{d}\right)=0(\forall c \neq d)$. If 
the relation between $P$ and $\mathbb{P}$ given in Eq. (12) holds, then $N P_{a b}$ of Eq. (21) is identical to $N P_{a b}^{\Pi}$ of Eq. (1) in Definition 1 for all choices of $a \neq b$ in $\mathscr{b}$.

Now that we have defined and discussed binary net preference probabilities in terms of random utilities, we can define majority rule in terms of random utilities as well, in a way that is consistent with the previous definition in terms of probabilities on preference relations.

Definition $3^{\prime}$. Given binary net probabilities $N P$ (derived from a family of jointly distributed random variables), define majority rule social welfare relations $\succeq$ and $>$ through, $\forall c, d \in \mathscr{C}$

$$
\begin{aligned}
& c \succeq d \Leftrightarrow N P_{c d} \geq 0, \\
& c \succeq d \Leftrightarrow N P_{c d}>0 .
\end{aligned}
$$

We say that $c$ has a weak majority over $d$ (including the possibility of a tie) whenever $c \succeq d$. We also say that $c$ has a strict majority over $d$ (excluding the possibility of a tie) whenever $c>d$.

Whenever Observation 1 or Observation 2 applies, we can see that Definition $3^{\prime}$ is the random utility counterpart of Definition 3 for probabilistic preferences and the two definitions yield identical social welfare relations. This reconciles the concept of net preference probability distributions with the general framework of random utility representations. Note that the very way we derive pairwise majorities from random utilities implicitly chooses a family of binary relations for the representation of preferences. We have covered the most natural cases, which correspond to linear, strict weak, semi-, interval, and strict partial orders, respectively. As a result of Observations 1 and 2 we can, without much loss of generality, from now on state our results only in terms of probabilities over (asymmetric) binary relations.

Remark. Note that the interval order and semiorder net preference probabilities in (19) and (20) cannot be obtained by simple substitution in (18) and, in that sense, are not special cases of (18). Rather, the concept of a (possibly variable) threshold is separate from the concept of dimension of a strict partial order. ${ }^{9}$ In other words, there exist two fundamentally different random utility representations that yield the identical probability distribution over semiorders or interval orders (and consequently the same net preference probability distribution and majority rule social welfare relations), depending on whether we view a semiorder or interval order (of dimension >1) as a ranking with a threshold of discrimination or as an intersection of two or more rankings (on separate dimensions). While this may appear mathematically awkward, it is psychologically intuitive, because the two random utility representations have fundamentally

\footnotetext{
${ }^{9}$ Incidentally, Bogart et al. (1976) have shown that a finite interval order can have arbitrarily high dimension, whereas Rabinovitch (1978) has shown that finite semiorders have dimension $\leq 3$, a result which was extended to arbitrary semiorders by Fishburn (1985) and Duggan (1999).
} 
different interpretations as psychological processes: in the first case, the respondent is unable to perfectly discriminate between every pair of alternatives, in the second case, the respondent is able to discriminate perfectly between every pair of alternatives, and does that even on two (or more) distinct dimensions. ${ }^{10}$

Thus, we can use random utility formulations and probabilistic preference formulations more or less interchangeably as long as we specify which set of binary relations we are referring to. Because of the above remark, we may, however, in some circumstance need to keep track of how we translate certain strict partial orders into real representations and vice versa.

\section{Generalizing the impartial culture and related cultures of indifference}

The impartial culture is usually defined as a uniform distribution over all linear orders (DeMeyer and Plott, 1970; Gehrlein and Fishburn, 1976) and occasionally defined as a uniform distribution over weak orders (Jones et al., 1995; Van Deemen, 1999). The most canonical generalization of the impartial culture to an arbitrary set of binary relations is therefore a uniform distribution over that set of relations. If all preference relations have equal probability, and if $B \in \mathscr{R} \Rightarrow B^{-1} \in \mathscr{R}$, then the probability that $a B b$ is equal to the probability that $b B a$, and thus the net probability $N P_{a b}$ is zero for all distinct $a, b \in \mathscr{C}$. The impartial culture is therefore a special case of a so-called culture of indifference (Tsetlin et al., 2002), by which we mean a culture in which all pairwise majority relationships are complete ties. ${ }^{11}$

As the following simple observation spells out, a culture of indifference (i.e. a completely tied social welfare order) requires very strong but also simple conditions.

\section{Observation 3.}

(i) Given a net probability distribution $N P$ (associated with some probability $P$ ) over asymmetric binary relations on any finite set $\mathscr{C}$, the majority social welfare order is a total tie if and only if $\forall a, b, c$ distinct in $\mathscr{C}$

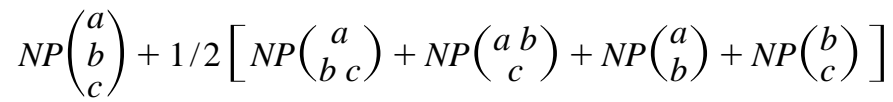

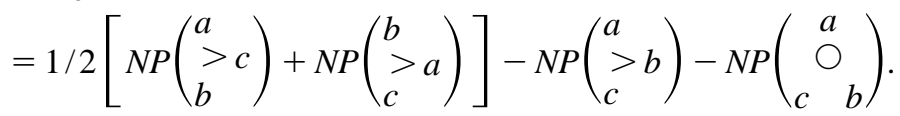

\footnotetext{
${ }^{10}$ This double interpretation has been there all along, of course, it has not been caused by the probabilistic framework.

${ }^{11}$ The typical use of the impartial and other cultures is to study social welfare relations in random samples drawn from such cultures. Of course, the majority preference relation in a random sample from the impartial culture need not be a complete tie.
} 
(ii) Given a net probability distribution $N P^{\mathscr{P O}}$ (associated with some probability $P^{\mathscr{P O}}$ ) over strict partial orders on any finite set $\mathscr{C}$, the majority social welfare order is a total tie if and only if $\forall a, b, c$ distinct in $\mathscr{C}$

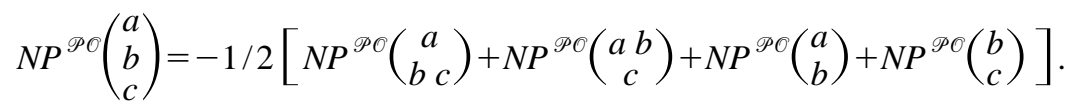

(iii) Given a net probability distribution $N P^{\mathscr{W O}}$ (associated with some probability $P^{\mathscr{W O}}$ ) over strict weak orders on any finite set $\mathscr{C}$, the majority social welfare order is a total tie if and only if $\forall m, n, k$ distinct in $\mathscr{C}$

$$
N P^{\mathscr{W O}}\left(\begin{array}{l}
m \\
n \\
k
\end{array}\right)=-1 / 2\left[N P^{\mathscr{W O}}\left(\begin{array}{c}
m \\
n k
\end{array}\right)+N P^{\mathscr{W O}}\left(\begin{array}{c}
m n \\
k
\end{array}\right)\right] .
$$

In particular, the following property has to consequently hold for any choice of distinct $a, b, c$ :

$$
N P^{\mathscr{W O}}\left(\begin{array}{l}
a \\
b \\
c
\end{array}\right)+N P^{\mathscr{W O}}\left(\begin{array}{l}
b \\
c \\
a
\end{array}\right)+N P^{\mathscr{W O}}\left(\begin{array}{l}
c \\
a \\
b
\end{array}\right)=0
$$

(iv) Given a net probability distribution $N P^{\Pi}$ (associated with some probability $P^{\Pi}$ ) over linear order preferences, the majority social welfare order is a total tie if and only if $\forall m, n, k$ distinct in $\mathscr{C}$

$$
N P^{\Pi}\left(\begin{array}{l}
m \\
n \\
k
\end{array}\right)=0 \text {. }
$$

\section{Conclusions}

By defining majority social welfare orders in terms of the theoretical primitives that are common to virtually all models of choice, rating or rankings, we provide a common ground for the theoretical and empirical analysis of majority rule within a panoply of basic and applied research paradigms. In an empirical companion paper (Regenwetter et al., 2002b) we show that the reconstruction of majority and other social welfare relations from ballot or survey data can critically depend on the underlying model of preferences or utilities. This suggests that anyone who analyses ballot or survey data needs to worry about the sensitivity or robustness of the results under variations or violations of the implicit or explicit model of preferences and utilities used in the analysis. Another strand of work also investigates the statistical issues in a sampling and inference framework (Tsetlin and Regenwetter, 2002).

\section{Acknowledgements}

We thank the National Science Foundation for funding this collaborative research through NSF grants SBR 97-30076 to Regenwetter and SBR 97-30578 to Grofman and 
Marley. The paper has also benefitted from interactions at Random Utility 2000, a conference funded via NSF grant SBR 98-18756 to Regenwetter and Pekeč. We thank Saša Pekeč, an anonymous referee and the editor for their helpful comments on earlier drafts. This research was carried out while the first author was a faculty member of the Decision Sciences group at the Fuqua School of Business, Duke University, which we thank for its valuable support. Marley was a fellow at the Hanse-Wissenschaftskolleg, Germany, during the paper's completion.

\section{Appendix A}

Proof of Theorem 1. Let

$$
\begin{aligned}
& x_{1}=N P\left(\begin{array}{l}
a \\
b \\
c
\end{array}\right) \quad x_{2}=N P\left(\begin{array}{l}
a \\
c \\
b
\end{array}\right) \quad x_{3}=N P\left(\begin{array}{l}
b \\
a \\
c
\end{array}\right) \\
& x_{4}=N P\left(\begin{array}{l}
a \\
b
\end{array}\right) \quad x_{5}=N P\left(\begin{array}{l}
a b \\
c
\end{array}\right) \quad x_{6}=N P\left(\begin{array}{c}
b \\
a c
\end{array}\right) \\
& x_{7}=N P\left(\begin{array}{l}
a \\
b
\end{array}\right) \quad x_{8}=N P\left(\begin{array}{l}
b \\
c
\end{array}\right) \quad x_{9}=N P\left(\begin{array}{l}
a \\
c
\end{array}\right) \\
& x_{10}=N P\left(\begin{array}{l}
\bigcirc \\
c
\end{array}\right) \\
& x_{11}=N P\left(\begin{array}{l}
b \\
>a \\
c
\end{array}\right) \quad x_{12}=N P\left(\begin{array}{l}
a \\
>b \\
c
\end{array}\right) \quad x_{13}=N P\left(\begin{array}{l}
a \\
>c \\
b
\end{array}\right) \\
& y_{1}=N P^{\Pi}\left(\begin{array}{l}
a \\
b \\
c
\end{array}\right) \quad y_{2}=N P^{\Pi(}\left(\begin{array}{l}
a \\
c \\
b
\end{array}\right) \quad y_{3}=N P^{\Pi(}\left(\begin{array}{l}
b \\
a \\
c
\end{array}\right) .
\end{aligned}
$$

Now suppose that (6) holds, i.e.

$$
\begin{aligned}
& y_{1}+y_{2}-y_{3}=N P_{a b}^{I I}=N P_{a b}=x_{1}+x_{2}-x_{3}+x_{4}-x_{6}+x_{7}+x_{10}-x_{11}+x_{12}, \\
& y_{1}-y_{2}+y_{3}=N P_{b c}^{I I}=N P_{b c}=x_{1}-x_{2}+x_{3}+x_{5}+x_{6}+x_{8}+x_{10}+x_{12}-x_{13}, \\
& -y_{1}-y_{2}-y_{3}=N P_{c a}^{I I}=N P_{c a}=-x_{1}-x_{2}-x_{3}-x_{4}-x_{5}-x_{9}+x_{10}-x_{11}-x_{13} .
\end{aligned}
$$

It follows that

$$
\begin{aligned}
& y_{1}=x_{1}+\frac{1}{2}\left(x_{4}+x_{5}+x_{7}+x_{8}-x_{11}-x_{13}\right)+x_{10}+x_{12}, \\
& y_{2}=x_{2}+\frac{1}{2}\left(x_{4}-x_{6}-x_{8}+x_{9}+x_{11}-x_{12}\right)-x_{10}+x_{13},
\end{aligned}
$$




$$
y_{3}=x_{3}+\frac{1}{2}\left(x_{5}+x_{6}-x_{7}+x_{9}-x_{12}+x_{13}\right)-x_{10}+x_{11},
$$

and thus (7) holds. Conversely, suppose that (7) holds. Then,

$$
\begin{aligned}
& N P_{a b}^{I I}=N P^{\Pi}\left(\begin{array}{l}
a \\
b \\
c
\end{array}\right)+N P^{\Pi}\left(\begin{array}{l}
a \\
c \\
b
\end{array}\right)+N P^{\Pi}\left(\begin{array}{l}
c \\
a \\
b
\end{array}\right)=y_{1}+y_{2}-y_{3}
\end{aligned}
$$

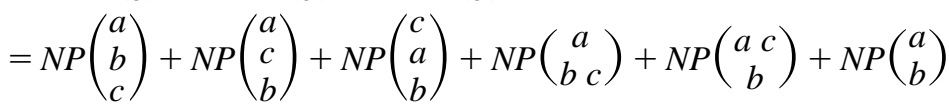

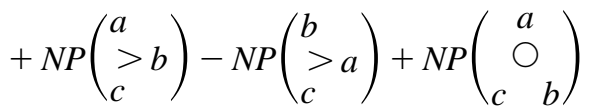

$$
\begin{aligned}
& =N P_{a b} \text {, }
\end{aligned}
$$

i.e. (6) holds. The rest of the theorem is immediate.

Proof of Theorem 4. The sets $\Pi^{k}(B)$ form a partition of $\Pi^{k}$ when we let $B$ vary over all of $\mathscr{P} O(k)$. Thus, for $B \neq B^{\prime}$ we have $\Pi^{k}(B) \cap \Pi^{k}\left(B^{\prime}\right)=\emptyset$ and the corresponding events entering the right-hand side of (16) are disjoint for such $B$ and $B^{\prime}$. It is also evident that all these events are well defined. We only need to check whether the probabilities sum to 1. Before we proceed, we introduce the following abbreviating notation:

$$
\begin{aligned}
& \Psi=\underset{\left(\pi_{1}, \pi_{2}, \ldots, \pi_{k}\right) \in \Pi^{k}(B)}{\cup} \bigcap_{i=1}^{k} \bigcap_{(c, d) \in \pi_{i}}\left(\boldsymbol{U}_{i, c}>\boldsymbol{U}_{i, d}\right), \\
& \Delta=\bigcap_{i=1}^{k} \bigcap_{(a, b) \in \mathscr{C}^{2}-I}\left(\boldsymbol{U}_{i, a} \neq \boldsymbol{U}_{i, b}\right),
\end{aligned}
$$

where $I$ denotes the identity, as noted before. We now prove that $\Psi=\Delta$ from which the theorem follows.

(i) To prove that $\Psi \subseteq \Delta$, consider an arbitrary sample point $\omega \in \Psi$. Then $\exists\left(\pi_{1}, \ldots, \pi_{k}\right) \in \Pi^{k}$ and $B \in \mathscr{P} \mathcal{O}(k)$ such that $B=\cap_{i=1}^{k} \pi_{i}$ and such that $\forall i$, $\forall(c, d) \in \pi_{i}$

$$
\boldsymbol{U}_{i, c}(\omega)>\boldsymbol{U}_{i, d}(\omega)
$$

Therefore, $\forall i, \forall(a, b) \in \mathscr{C}^{2}-I$ we have

$$
\boldsymbol{U}_{i, a}(\omega) \neq \boldsymbol{U}_{i, b}(\omega)
$$

and thus $\omega \in \Delta$.

(ii) To prove that $\Delta \subseteq \Psi$, consider an arbitrary sample point $\omega \in \Delta$. Then, $\forall i$, $\forall(a, b) \in \mathscr{C}^{2}-I$ we have

$$
\boldsymbol{U}_{i, a}(\omega) \neq \boldsymbol{U}_{i, b}(\omega) .
$$

Now, for each $i$ define

$$
\pi_{i}=\left\{(c, d) \in \mathscr{C}^{2} \mid \boldsymbol{U}_{i, c}(\omega)>\boldsymbol{U}_{i, d}(\omega)\right\} .
$$


Clearly, each $\pi_{i}$ is a linear order. Finally, the relation $B=\cap_{i=1}^{k} \pi_{i}$ is a strict partial order of dimension at most $k$. Since, furthermore, $\forall i, \forall(c, d) \in \pi_{i}$ it is the case that

$$
\boldsymbol{U}_{i, c}(\omega)>\boldsymbol{U}_{i, d}(\omega)
$$

we must conclude that $\omega \in \Psi$.

Therefore, in order for the probabilities to sum to one, we verify that the following equivalent conditio ns hold

$$
\begin{aligned}
\sum_{B \in \mathscr{P} O(k)} P(B)=1 & \Leftrightarrow \mathbb{P}(\Psi)=1 \\
& \Leftrightarrow \mathbb{P}(\Delta)=1 \\
& \Leftrightarrow \mathbb{P}\left(\bigcap_{i=1}^{k} \bigcap_{(c, d) \in \mathscr{C}^{2}-I}\left(\boldsymbol{U}_{i, c} \neq \boldsymbol{U}_{i, d}\right)\right)=1, \\
& \Leftrightarrow \mathbb{P}\left(\bigcup_{i=1}^{k} \bigcup_{(c, d) \in \mathscr{C}^{2}-I}\left(\boldsymbol{U}_{i, c}=\boldsymbol{U}_{i, d}\right)\right)=0, \\
& \Leftrightarrow \sum_{i=1}^{k} \sum_{c \neq d} \mathbb{P}\left(\boldsymbol{U}_{i, c}=\boldsymbol{U}_{i, d}\right)=0, \\
& \Leftrightarrow \mathbb{P}\left(\boldsymbol{U}_{i, c}=\boldsymbol{U}_{i, d}\right)=0, \quad \forall i \in\{1, \ldots, k\}, \forall(c, d) \in \mathscr{C}^{2}-I .
\end{aligned}
$$

Proof of Theorem 5. To prove that $(1) \Rightarrow(2)$, notice that as stated at the beginning of the proof of Theorem 4, we have $\Pi^{k}(B) \cap \Pi^{k}\left(B^{\prime}\right)=\emptyset$ for $B \neq B^{\prime}$. Thus, the corresponding events on the left-hand side of Eq. (17) are disjoint for such $B$ and $B^{\prime}$. From that, (2) follows immediately.

To prove $(2) \Rightarrow(1)$, suppose that there are $q$ many atoms $\left\{A_{1}\right\}, \ldots,\left\{A_{q}\right\}$ in $\langle\mathscr{P} \mathscr{O}(k)$, $\left.2^{\mathscr{P} O(k)}, P\right\rangle$. By Assumption (2) we can partition $\Omega$ into $O_{1}, \ldots, O_{q} \in \sigma$. Consider $j \in\{1, \ldots, q\}$. Then there exists $\pi_{i}, i=1, \ldots, k$, such that $A_{j}=\cap_{i=1}^{k} \pi_{i}$. Now, $\forall \omega \in O_{j}, \forall(c, d) \in \mathscr{C}^{2}, \forall i=1, \ldots, k$, choose values for $\boldsymbol{U}_{i, c}(\omega), \boldsymbol{U}_{i, d}(\omega)$ in any way such that

$$
\boldsymbol{U}_{i, c}(\omega)>\boldsymbol{U}_{i, d}(\omega) \Leftrightarrow(c, d) \in \pi_{i}
$$

By construction, $\mathbb{P}\left(O_{j}\right)=P\left(A_{j}\right)$. It is straightforward to extend the probability measure $\mathbb{P}$ to all of $\sigma$ : pick any probability measure $\tilde{\mathbb{P}}$ on $\langle\Omega, \sigma\rangle$ such that $\tilde{\mathbb{P}}\left(O_{j}\right) \neq 0$, for $j=1, \ldots$, $k$ and for each $X \in \sigma$ define

$$
\mathbb{P}(X)=\sum_{j=1}^{q} \frac{\tilde{\mathbb{P}}\left(X \cap O_{j}\right)}{\tilde{\mathbb{P}}\left(O_{j}\right)}
$$

As a result of this construction, $\boldsymbol{U}$ is a well-defined family of jointly distributed random variables on the given sample space, and $\boldsymbol{U}$ satisfies (17). 


\section{References}

Arrow, K.J., 1951. Social Choice and Individual Values. Wiley, New York.

Barberá, S., 1979. Majority and positional voting in a probabilistic framework. The Review of Economic Studies 46, 379-389.

Barberá, S., Pattanaik, P.K., 1986. Falmagne and the rationalizability of stochastic choices in terms of random orderings. Econometrica 54, 707-715.

Ben-Akiva, M.B., Lerman, S.R., 1985. Discrete Choice Analysis: Theory and Applications to Travel Demand. MIT Press, Cambridge, MA.

Block, H.D., Marschak, J., 1960. Random orderings and stochastic theories of responses. In: Olkin, I., Ghurye, S., Hoeffding, H., Madow, W., Mann, H. (Eds.), Contributions to Probability and Statistics. Stanford University Press, Stanford, pp. 97-132.

Bogart, K., Rabinovitch, I., Trotter, W.T.J., 1976. A bound on the dimension of interval orders. Journal of Combinatorial Theory 21, 319-328.

Brady, H., Ansolabehere, S., 1989. The nature of utility functions in mass publics. American Political Science Review 83 (1), 143-163.

Coughlin, P., 1992. Probabilistic Voting Theory. Cambridge University Press, New York.

Critchlow, D.E., Fligner, M.A., Verducci, J.S., 1991. Probability models on rankings. Journal of Mathematical Psychology 35, 294-318.

Critchlow, D.E., Fligner, M.A., Verducci, J.S. (Eds.), 1993. Probability Models and Statistical Analyses for Ranking Data. Springer, New York.

DeMeyer, F., Plott, C.R., 1970. The probability of a cyclical majority. Econometrica 38, 345-354.

Duggan, J., 1999. A general extension theorem for binary relations. Journal of Economic Theory 86, 1-16.

Enelow, J., Hinich, M.J., 1984. Probabilistic voting and the importance of centrist ideologies in democratic elections. Journal of Politics 46, 459-478.

Enelow, J., Hinich, M.J., 1989. A general probabilistic model of spatial voting. Public Choice 61, 101-114.

Falmagne, J.-C., 1978. A representation theorem for finite random scale systems. Journal of Mathematical Psychology 18, 52-72.

Falmagne, J.-C., 1997. Stochastic token theory. Journal of Mathematical Psychology 41, 129-143.

Falmagne, J.-C., Regenwetter, M., 1996. Random utility models for approval voting. Journal of Mathematical Psychology 40, 152-159.

Feld, S., Grofman, B., 1986. Partial single-peakedness: an extension and clarification. Public Choice 51, 71-80.

Fishburn, P.C., 1970. Intransitive indifference in preference theory. Journal of Mathematical Psychology 7, 207-228.

Fishburn, P.C., 1975. A probabilistic model of social choice: comment. Review of Economic Studies 42 , 297-301.

Fishburn, P.C., 1984. Probabilistic social choice based on simple voting comparisons. Review of Economic Studies 51, 683-692.

Fishburn, P.C., 1985. Interval Orders and Interval Graphs. Wiley, New York.

Fishburn, P.C., 1992. Induced binary probabilities and the linear ordering polytope: a status report. Mathematical Social Sciences 23, 67-80.

Fishburn, P.C., 1998. Stochastic utility. In: Barberá, S., Hammond, P.J., Seidl, C. (Eds.), Handbook of Utility Theory. Kluwer, Dordrecht, pp. 273-318.

Fishburn, P.C., Gehrlein, W.V., 1977. Towards a theory of elections with probabilistic preferences. Econometrica 45, 1907-1924.

Gaertner, W., Heinecke, A., 1978. Cyclically mixed preferences-a necessary and sufficient condition for transitivity of the social preference relation. In: Gottinger, H.W., Leinfellner, W. (Eds.), Decision Theory and Social Ethics. Reidel, The Netherlands, pp. 169-185.

Gehrlein, W.V., 1989. The probability of intransitivity of pairwise comparisons in individual preference. Mathematical Social Sciences 17, 67-75.

Gehrlein, W.V., Fishburn, P.C., 1976. The probability of the paradox of voting: a computable solution. Journal of Economic Theory 13, 14-25. 
Grandmont, J.-M., 1978. Intermediate preferences and the majority rule. Econometrica 46, 317-330.

Heyer, D., Niederée, R., 1989. Elements of a model-theoretic framework for probabilistic measurement. In: Roskam, E.E. (Ed.), Mathematical Psychology in Progress. Springer, Berlin, pp. 99-112.

Heyer, D., Niederée, R., 1992. Generalizing the concept of binary choice systems induced by rankings: one way of probabilizing deterministic measurement structures. Mathematical Social Sciences 23, 31-44.

Intriligator, M.D., 1973. A probabilistic model of social choice. Review of Economic Studies 40, 552-560.

Joe, H., 1997. Multivariate Models and Dependence Concepts. Chapman and Hall, London, UK.

Jones, B., Radcliff, B., Taber, C., Timpone, R., 1995. Condorcet winners and the paradox of voting: probability calculations for weak preference orders. The American Political Science Review 89 (1), $137-144$.

Kahneman, D., Tversky, A., 1979. Prospect theory: an analysis of decision under risk. Econometrica 47, 263-291.

Koppen, M., 1995. Random utility representation of binary choice probabilities: critical graphs yielding critical necessary conditions. Journal of Mathematical Psychology 39, 21-39.

Krantz, D.H., Luce, R.D., Suppes, P., Tversky, A., 1971. In: Foundations of Measurement, Vol. 1. Academic Press, San Diego.

Loomes, G., Sugden, R., 1982. Regret theory-an alternative theory of rational choice under uncertainty. Economic Journal 92, 805-824.

Luce, R.D., 1956. Semiorders and a theory of utility discrimination. Econometrica 26, 178-191.

Luce, R.D., 1959. Individual Choice Behavior: A Theoretical Analysis. John Wiley, New York.

Luce, R.D., Suppes, P., 1965. Preference, utility and subjective probability. In: Luce, R.D., Bush, R.R., Galanter, E. (Eds.). Handbook of Mathematical Psychology, Vol. III. Wiley, New York, pp. 249-410.

Luce, R.D., von Winterfeldt, D., 1994. What common ground exists for descriptive, prescriptive, and normative utility theories? Management Science 40, 263-279.

Mallows, C.L., 1957. Non-null ranking models I. Biometrika 44, 114-130.

Marley, A.A.J., 1991. Context dependent probabilistic choice models based on measures of binary advantage. Mathematical Social Sciences 21, 201-218.

Marschak, J., 1960. Binary-choice constraints and random utility indicators. In: Arrow, K.J., Karlin, S., Suppes, P. (Eds.), Proceedings of the First Stanford Symposium on Mathematical Methods in the Social Sciences, 1959. Stanford University Press, Stanford, CA, pp. 312-329.

McFadden, D., 1991. Advances in computation, statistical methods, and testing of discrete choice models. Marketing Letters 2, 215-229.

McFadden, D., 1998. Specification of econometric models. Econometrica, in press.

Niederée, R., Heyer, D., 1997. Generalized random utility models and the representational theory of measurement: a conceptual link. In: Marley, A.A.J. (Ed.), Choice, Decision and Measurement: Essays in Honor of R. Duncan Luce. Lawrence Erlbaum, Mahwah, NJ, pp. 155-189.

Rabinovitch, I., 1978. The dimension of semiorders. Journal of Combinatorial Theory 25, 50-61.

Regenwetter, M., 1996. Random utility representations of finite m-ary relations. Journal of Mathematical Psychology 40, 219-234.

Regenwetter, M., 1997. Probabilistic preferences and topset voting. Mathematical Social Sciences 34, 91-105.

Regenwetter, M., Adams, J., Grofman, B., 2002a. On the Sample Condorcet efficiency of majority rule: an alternative view of majority cycles and social homogeneity. Theory and Decision, conditionally accepted pending minor revision.

Regenwetter, M., Falmagne, J.-C., Grofman, B., 1999. A stochastic model of preference change and its application to 1992 presidential election panel data. Psychological Review 106, 362-384.

Regenwetter, M., Grofman, B., 1998a. Approval voting, Borda winners and Condorcet winners: evidence from seven elections. Management Science 44, 520-533.

Regenwetter, M., Grofman, B., 1998b. Choosing subsets: a size-independent probabilistic model and the quest for a social welfare ordering. Social Choice and Welfare 15, 423-443.

Regenwetter, M., Grofman, B., Marley, A., 2002b. On the model dependence of majority preferences reconstructed from ballot or survey data. Mathematical Social Sciences 43/3, 453-468.

Regenwetter, M., Marley, A.A.J., 2001. Random relations, random utilities, and random functions. Journal of Mathematical Psychology 45, 864-912.

Roberts, F.S., 1979. Measurement Theory. Addison-Wesley, London. 
Saari, D.G., 1995. Basic Geometry of Voting. Springer Verlag, Berlin.

Schofield, N., Tovey, C., 1992. Probability and convergence for supra-majority rule with euclidean preferences. Mathematical Computer Modelling 16, 41-58.

Suck, R., 1992. Geometric and combinatorial properties of the polytope of binary choice probabilities. Mathematical Social Sciences 23, 81-102.

Suck, R., 1995. Random utility representations based on semiorders, interval orders, and partial orders. Manuscript at Universität Osnabrück.

Tangian, A., 2000. Unlikelihood of Condorcet's paradox in a large society. Social Choice and Welfare 17, $337-365$.

Thurstone, L.L., 1927a. A law of comparative judgement. Psychological Review 34, 273-286.

Thurstone, L.L., 1927b. Three psychophysical laws. Psychophysical Review 34, 424-432.

Trotter, W.T., 1992. Combinatorics and Partially Ordered Sets. Johns Hopkins, London.

Tsetlin, I., Regenwetter, M., 2002. On the probability of correct or incorrect majority preference relations. Social Choice and Welfare, in press.

Tsetlin, I., Regenwetter, M., Grofman, B., 2002. The impartial culture maximizes the probability of majority cycles. Social Choice and Welfare, in press.

Tversky, A., 1969. Intransitivity of preferences. Psychological Review 76, 31-48.

Tversky, A., Kahneman, D., 1986. Rational choice and the framing of decisions. Journal of Business 59, 251-278.

Van Deemen, A., 1999. The probability of the paradox of voting for weak preference orderings. Social Choice and Welfare 16, 171-182.

Vila, X., 1998. On the intransitivity of preferences consistent with similarity relations. Journal of Economic Theory 79, 281-287. 